Aggarwal H.K. ${ }^{1}$, Jain D. ${ }^{2}$, Chhabra P. ${ }^{2}$

\title{
Tropical Diseases Nephropathy - An Underemphasized Comorbidity
}

${ }^{1}$ Department of Medicine IV and Registrar, Pt. B.D. Sharma University of Health Sciences, ROHTAK-124001(Haryana)

${ }^{2}$ Department of Medicine, Pt. B.D. Sharma University of Health Sciences, ROHTAK-124001(Haryana)

\begin{abstract}
The tropical areas are a home to a number of diseases because of the hot \& humid climate, and lack of health care facilities. Tropical Nephropathies are a major health problem and a matter of great concern. The main causes of kidney injury in tropics are due to bacterial, viral or parasitic infections like malaria, dengue, leptospirosis, scrub typhus, acute gastroenteritis, tuberculosis, leprosy and toxic envenomations like snake bite. Renal injury associated with tropical infections has a wide spectrum of presentations ranging from acute kidney injury to chronic kidney disease. Renal involvement in infectious diseases by one or other mechanism is a definite cause of increased morbidity and mortality. Proper understanding of these diseases, early diagnosis, patient education and improved health care facilities will lead to a better outcome and improved survival. This article reviews the various tropical infections causing kidney injury including pathophysiology and line of management.
\end{abstract}

Keywords: kidney injury, infectious diseases, malaria, tropical infections

\section{Jain Deepak}

Department of Medicine, PGIMS, Rohtak-124001 (Haryana), India

email : jaindeepakdr@gmail.com phone: +91 9416147887

\section{Introduction}

The Tropics also known as tropical or torrid zones are the region of the Earth near to the equator and are geographically limited by the tropics of cancer and Capricorn. Hot and humid climate characterises these areas encompassing around almost one third of earth's landmass [1]. Hot and humid climate, poverty, overcrowding, unhygienic conditions, and poor access to health care services are the contributing factors for a vast number of health issues seen in the tropics.

Immigration and increasing travel have made once neglected tropical diseases relevant for the whole globe. Kidney Injury in the tropical areas has been always an important health related concern. Tropical Nephrology is a matter of concern in view of challenging environmental and socioeconomic factors. The pathophysiology and treatment modalities of renal injury are similar in tropics and temperate areas inspite of differences in the epidemiology. Acute kidney injury (AKI) in tropics is influenced by number of factors like infections, use of unsafe drinking water, exposure to environmental toxins, poverty, inadequate sanitation and envenomation by snakes and arthropods. High temperature and humidity in tropics causes peripheral vasodilatation and sweating leading on to dehydration and decreased intravascular 
volume which contributes to AKI. Kidney injury in tropics also depends on the seasonal variation as there is an increased incidence of infections and snake bites during and immediately after rainy seasons. Heavy rainfall creates a favourable environment for survival of organisms causing and transmitting infections like malaria, dengue etc. $[2,3]$.

Infectious diseases like malaria, dengue, leptospirosis, diarrhoea, scrub typhus, tuberculosis, leprosy and toxic envenomation like snake bite contribute to majority cases of kidney injury in tropics. On the other hand developed countries exhibit different spectrum of causes of renal injury like trauma, industrial accidents, drugs, heart diseases and renal transplantation rejection. in the In tropical zone kidney injury occurs at much younger age in comparison to temperate zone. In India the mean age affected with renal injury is 37.1 years, while in temperate zone the mean age is higher being around 72 years. The most common cause in tropical areas is community acquired infections causing acute tubular necrosis (ATN). A recent study in South India from a large tertiary care hospital showed that $41.1 \%$ of patients with tropical infections have AKI and scrub typhus, malaria, salmonellosis, dengue and leptospirosis are the most common implicating infections[4]. Over the past three decades, several studies conducted in India on patients with AKI have shown the percentage of medical, obstetrica and surgical conditions resulting in acute renal failure (ARF) as $60 \%, 15 \%$ and $25 \%$; and $88 \%, 9 \%$ and 3.4 ; respectively $[5,6]$. In India over $40 \%$ of all causes of AKI are due to diarrheal diseases, snake bites, intravascular hemolysis due to G6PD deficiency, and copper sulphate poisoning [6].

The renal involvement in tropical diseases has a large spectrum ranging from asymptomatic proteinuria or urinary sediment abnormalities to fatal acute renal failure requiring renal transplantation. Direct infection of the kidneys and urinary tract or systemic effects of infection leads to renal derangement. Glomerular, microvascular, and tubulointerstitial lesions are the most common renal pathologies and lesions depend upon the etiological infectious disease [7]. Although these infections most commonly cause AKI, the residual damage which may be subclinical can lead to chronic kidney disease.
Despite increasing awareness and expanding health providing system, the Infectious tropical nephropathies associated with various bacterial, viral and parasitic infections being an important cause of morbidity and mortality are posing challenges. Various infections causing tropical nephropathy are as under.

\section{Malaria}

Malaria is a protozoal disease of extreme epidemiological concern because of its greater prevalence in tropical areas. Around 103 endemic countries are affected by malaria resulting in 1-3 million deaths per year [8]. Malaria caused by $P$. falciparum and P. malariae most frequently affect kidneys. [9]. AKI is a very devastating manifestation of malaria reported in about 3\% malarial cases. Severe P. falciparum malaria has a high incidence of renal involvement seen in around $60 \%$ patients.

There have been different pathophysiological mechanisms behind AKI seen in malaria. Various proposed mechanisms are decreased intravascular volume, mechanical obstruction by infected erythrocytes (cytoadherence), arterial vasodilatation mediated by cytokines and nitric oxide, resistance to vasoactive hormones, and decreased ATP synthesis due to cytopathic hypoxia[10]. The parasite inhabitates erythrocytes and alters their morphology and metabolism. It leads to formation of "knobs" on the red blood cell's surface. Infected cells adhere to the vascular endothelium and uninfected RBCs to form rosettes. This promotes cytoadherence between RBCs, platelets and endothelium leading on to microcirculatory failure and organ dysfunction. Immune system activation also leads to endothelial injury and immune complex deposition. Proinflammatory cytokines, reactive oxygen species and vasoactive mediators lead to hemodynamic changes causing diminished renal blood flow and glomerular filtration rate. Ischemic and hypoxia therefore leads to AKI in malaria and usually seen 
in patients with heavy parasitemia [10]. Hemolysis in patients with glucose-6-phosphate dehydrogenase enzyme due to drugs like quinine and primaquine further aggravates AKI. ATN is the commonest cause of kidney injury in malaria and other important causes are acute tubulointerstitial nephritis, diffuse proliferative and mesangioproliferative glomerulonephritis

Acute malarial nephropathy and chronic malarial nephropathy are two major clinical syndromes associated with kidney involvement in malaria [11]. Acute malarial nephropathy presents as AKI seen in falciparum malaria while quartan or chronic malarial nephropathy mostly caused by P. malariae. is glomerulopathy usually seen in children. However glomerular involvement in the form of nephritic to nephritic syndrome has also been reported in $\mathrm{P}$. falciparum infection. $\mathrm{P}$. falciparum infection causes acute fulminant disease known as complicated malaria and manifestations include anaemia, gastroenterities, jaundice, coagulation defect, acute respiratory failure and coma along with acute nephropathy. Jaundice occurs in malaria with AKI and is reported in more than $75 \%$ of cases [12]. Most common electrolyte disturbances include hyperkalemia, hypokalemia, hyponatremia, hypocalcaemia and hypophosphatemia 13].

Any patient from the tropics with fever and one or more of the common malarial manifestations, i.e., altered sensorium, AKI, anaemia and jaundice should be suspected as malaria. Non oliguric AKI is seen in around $50-75 \%$ of patients and usually occurs by the end of first week 75[13]. Nephritic or nephrotic syndrome a clinical manifestation particularly of chronic malarial nephropathy presents with edema after a few weeks of fever and urinalysis showing proteinuria.

Peripheral blood smears stained with Giemsa stain identifies sexual forms of the parasite and is the most common modality used for the laboratory diagnosis. Rapid Diagnostic immunological tests are currently available. Urinalysis can reveal hyaline \& cellular casts, micro albuminuria and proteinuria [14]. Renal histology shows ATN predominantly affecting the distal tubules. Tubular changes like haemosiderin granular deposits, haemoglobin casts, interstitial edema and mononuclear cell interstitial infiltrates are the most common histological changes in kidneys. Peritubular capillaries may be packed with parasitized red blood cells in patients with heavy parasitaemia. In some patients glomerulonephritis and interstitial nephritis have also been seen.

Malarial AKI has a mortality rate that varies between $15 \%$ and $50 \%$ [10]. Intravenous quinine and artemisinin compounds are the first line of drugs used in the treatment [15]. Haemodialysis has a very good outcome if initiated early in the course of treatment. $50 \%$ to $80 \%$ of patients require dialysis [16]. In contrast chronic malarial nephropathy usually progress to end stage renal disease and is non responsive to antimalarial therapy or immunosuppressive therapy.

\section{Dengue}

Dengue is a viral haemorrhagic fever caused by Dengue Flavivirus is and transmitted by Aedes aegypti mosquitoes. $70 \%$ of the total global burden of this disease is shared by South East Asia and Western Pacific regions in tropics. The incidence and prevalence of dengue has been on a increasing trend due to factors such as climate changes, demographic factors, ecosystem modifications, uncontrolled and unplanned urbanization and population migration [17].

Undifferentiated fever, dengue fever, dengue haemorrhagic fever (DHF) and dengue shock syndrome (DSS) are different manifestations of dengue virus infection. This disease can be severe in the form DHF and DSS which are characterized by fever, haemorrhagic phenomena, low platelet count, plasma leakage (increased haematocrit, serositis and hypoalbuminemia), decreased sensorium, respiratory distress, myocarditis, shock and can be fatal leading to death[17]. Kidney involvement is a well-known complication of dengue fever and can manifest as AKI, glomerulonephritis or haemolytic uraemic syndrome [18]. DHF and DSS are more commonly associated with AKI which acts as an independent predictor of mortality [19]. The reported frequency of 
dengue associated AKI is extremely variable, ranging from $1 \%$ to approximately $30 \%$. AKI was reported in $10.8 \%$ of patients with dengue infection in recent study from India [20]. The main causes of AKI include dengue shock syndrome, haemorrhagic shock due to bleeding and ATN due to rhabdomyolysis. Renal failure can also occur due to the direct involvement of the kidneys without shock and rhabdomyolysis [21]. Diagnosis mainly depends upon the serological tests available. The mainstay of management is prompt identification of complications and supportive therapy because dengue fever has no specific treatment and therefore there are no specific drugs available. Use of drugs like aspirin and NSAIDs should be avoided.

\section{Leptospirosis}

Leptospirosis is a zoonotic disease caused by spirochetes of the genus Leptospira and has a worldwide distribution. Leptospira has more than 200 pathogenic serovars mostly found in tropical and subtropical areas. Leptospirosis is included in the list of reemerging infectious diseases by WHO in both developed and developing countries [22]. Tropical areas has an approximately 100 times incidence of this disease as compared to temperate areas due to humid climate of tropics. Leptospirosis is reported from India, Southeast Asia, Africa and South America. Contact of abraded skin or mucous membrane with infected tissue or urine of animal host or indirectly through contaminated water, soil or vegetation are the various modes of human infection. There is a sharp increase in incidence of Leptospirosis during or soon after rainy season and flooding, but it can occur all around the year.

Leptospirosis has a broad spectrum of manifestations ranging from asymptomatic condition to a severe multisystem disease depending upon the serotype and geographical area. Leptospirosis can either present as a febrile, auto-limited disease or as Weil's syndrome with triad of jaundice, haemorrhage and renal failure. It is biphasic and has two stages.
Fever lasting from 3-10 days characterises the acute leptospiremic phase and renal injury occurring after a relative asymptomatic period of 1-3 days occurs in the immune phase. AKI associated with leptospirosis is non oliguric and usually accompanied by hypokalemia and may involve multiple organs like liver and lungs. Severe jaundice is frequently seen in leptospirosis leading to hastening of development and increased severity of AKI. In severe leptospirosis incidence of renal involvement varies from $40 \%$ to $60 \%$ [23].

Various factors are responsible for AKI seen in severe leptospirosis. Bacterial invasion, direct toxicity of bacterial products, inflammation and haemodynamic changes are the various proposed pathogenic mechanisms[24]. Tubulointerstitial nephritis due to direct infection of the kidneys is the predominant mechanism of renal involvement. Lesions are associated with physical presence of organism as shown by various experimental studies. Polyuria and enhanced natriuresis occurs due to proximal tubule damage and vasopressin resistance in collecting duct thus leading to reduced proximal sodium reabsorption and increase free water clearance. A prospective study demonstrated the presence of a proximal tubule defect in leptospirosis associated AKI [25].

Leptospirosis can be diagnosed by direct examination of blood for leptospira, or by its culture in the initial febrile period. Leptospira may be found and cultured from urine or a blood serology can be used during the immune phase. The macroscopic agglutination test may also be used as a screening test. Interstitial edema and infiltration with mononuclear cells and few eosinophils are the pathological findings seen in renal biopsy. Glomeruli are usually spared and ATN primarily affecting the proximal tubules is present.

The treatment of severe leptospirosis mainly involves the use of antibiotics and hemodialysis. Antimicrobial drugs effective in leptospirosis like crystalline penicillin or doxycycline shorten the duration of illness and thus reducing hospital stay. Intravenous ceftriaxone or cefotaxime are equally efficacious as compared to penicillin [26]. Serum creatinine normalisation depends on the severity of kidney involvement and usually normalises by the 
fourth to eighth day of symptomatic disease. Complete recovery of glomerular filtration rate, proximal sodium reabsorption, tubular hydrogen generation and fractional potassium excretion, take place by the third month of follow up. In some patients concentration defect can persist for longer periods. Antibiotics use during the first week of infection reduces symptoms and AKI severity. Mortality can be reduced by early dialysis and management of renal injury.

\section{Scrub Typhus}

Scrub typhus is an acute febrile illness and zoonosis caused by orientia tsutsugamushi. Mode of transmission to humans is through the bite of the larva of trombiculid mites. This disease is endemic to a part of the world known as the "tsutsugamushi triangle", extending from northern Japan and far eastern Russia in the north, to the territories around the Solomon Sea into northern Australia in the south, and to Pakistan and Afghanistan in the west [27]. It is widely spread all over India and has been reported in several parts of the country [28].

Scrub Typhus mainly affects outside working people as they are exposed to shrubs and vegetation, on which the vector thrives, mostly during and after the rainy season,. Presence of a primary papular lesion which enlarges undergoes central necrosis, and crusts to form a flat black eschar is a classical presentation of the disease. This is associated with regional and later generalized lymphadenopathy. The spectrum of the clinical severity of scrub typhus ranges from mild to severe with fatal complications such as meningoencephalitis, pneumonitis, acute respiratory distress syndrome, AKI, myocarditis, disseminated intravascular coagulation (DIC), and septic shock [29]. The reported incidence of renal failure caused by scrub typhus varies from $10.5 \%$ to $42.6 \%$. In the Indian subcontinent AKI has been reported in about 30 to $60 \%$ of the patients of scrub typhus.

There are various explanations behind the pathophysiology of AKI in scrub typhus. Vasculitis of the small blood vessels, DIC, hypovolemia and shock causing renal hypoperfusion, injury to vascular endothelium are main mechanisms of renal involvement [30]. Tubulointerstitial nephritis caused by direct invasion of the renal parenchyma by the microorganism may also cause renal failure. Diagnosis of scrub typhus can be made by a number of tests like Weil Felix test, ELISA, Western Blot along with renal biopsy. Indirect Immunofluorescence remains the golden standard.

Renal failure caused by scrub typhus is commonly known to be reversible with the appropriate antibiotic therapy, and rarely requires maintenance hemodialysis. Doxycycline and azithromycin are the most commonly used antimicrobials effective in treatment. Early diagnosis and appropriate treatment improves the overall prognosis of the disease.

\section{Tuberculosis}

Tuberculosis (TB) is a systemic disease caused by Mycobacterium tuberculosis and has a high prevalence in developing parts of the world. Its most common manifestation is as pulmonary tuberculosis while genitourinary disease is the second most common form of non-pulmonary tuberculosis after tubercular lymphadenitis. Infection with human immunodeficiency virus, and immunosuppression due to increased number of organ transplantations have lead on to increased number of patients diseased with extra pulmonary TB.

The most common clinical presentation is pulmonary cavitation, usually accompanied by productive cough, fever, weight loss and night sweating. The widespread seeding of tubercular bacilli leads to lesions in other locations like lymph nodes, pleura and the urogenital tract ${ }^{9}$. Patients with tuberculosis of the urinary tract usually present with lower urinary symptoms typical of conventional bacterial cystitis thus masquerading genitourinary TB. Loss of kidney function, obstructive uropathy and bladder defects are sequelae seen in advanced 
disease [31].

Urogenital tuberculosis can occur in various forms like classical renal tuberculosis,,glomerular disease and interstitial nephritis progressing to end stage renal disease, dialysis and transplantation associated tuberculosis and genital tuberculosis (most commonly affecting the epididymis and prostate) [32]. Secondary amyloidosis with renal involvement is important late complication of tuberculosis and usually present as nephrotic range proteinuria. Urogenital tuberculosis is always secondary to a respiratory inoculation, which may be sometimes be clinically unapparent. The kidneys are initially involved and then the lesions advance distally to the ureters, bladder and testicles. Urine examination shows proteinuria, pyuria or haematuria. The urine cultures are negative inspite of the consistent pyuria. Urogenital tuberculosis can be diagnosed by mycobacterium identification and culture but it takes a longer time. Polymerase chain reaction (PCR) techniques, such as the direct Gen-Probe test are a reliable and rapid diagnostic modality. In early disease, intravenous urography shows calyceal distortion and detects changes even in a single calyx. Ultrasonography, computerized tomography and magnetic nuclear resonance imaging reveal grossly distorted ureters, with alternating stenotic and dilated areas and a fibrosed bladder [33]. Cystoscopy with biopsy can be used to visualise and sample bladder lesions.

Kidney biopsies mostly show a granuloma with an area of central caseous necrosis and tubulointerstitial inflammation [34]. Granuloma may unveil acid-fast bacilli suggestive of tuberculosis. Urogenital tuberculosis treatment mainly includes a complete course of antitubercular therapy. Advanced obstructive lesions may require surgical intervention.

\section{Diarrhoeal Diseases}

Diarrhoeal diseases are widely prevalent in the tropical developing countries due to factors like lack of clean water supply and low socio economic conditions. The causative organism can either be a bacteria virus or a parasite. Causative organisms include bacterias including Escherichia coli, Campylobacter, Salmonella, Shigella \& Vibrio Cholera, viruses like Rotavirus, Norovirus, Adenovirus \& Astrovirus and parasites like Giardia lambia, Entamoeba histolytica \& Cryptosporidium. Most cases of AKI in children in India are due to diarrhoeal diseases [35]. Onset of summer months increases the incidence of these diseases reaching maximum frequency during the rainy season. Acute tubular necrosis is the commonest cause of renal injury mainly due to hypovolemia and shock. Oliguric renal failure and metabolic acidosis out of proportion to renal parameters is usually seen. This is because of the loss of bicarbonate in the diarrhoeal fluid. Management approach includes adequate hydration and correction for intra and extravascular volume deficit produced by fluid losses in diarrhoea. Treatment options depend upon the severity of dehydration and general condition of the patient. For patients with mild to moderate dehydration and no vomiting, oral rehydration therapy is recommended. The last decade has seen an decreased incidence of AKI due to diarrhoeal diseases particularly due to early use of oral rehydration therapy and an improved living standard[35]. In severely dehydrated patients with shock, persistent vomiting or paralytic ileus intravenous dehydration with Ringer's lactate is necessary. Potassium supplementation may be required for prevention of life threatening cardiac dysrhythmias. because hypokalemia can worsen following correction of metabolic acidosis. Rarely there is need for hemodialysis in fulminating cases of kidney injury with diarrhoea. 
Some other bacterial, fungal, parasitic and viral infectious diseases are needed to be mentioned which are associated with significant renal involvement. Renal injury in HIV infected patients in the form of acute tubular necrosis is usually secondary to sepsis, hypotension, dehydration and nephrotoxins. Renal involvement may manifest as acute kidney injury, HIV associated nephropathy (HIVAN), HIV related immune complex diseases, nephropathy secondary to ART and diseases related to common comorbidities [36]. Increased HIV viral loads, reduced CD4 cell counts, hepatitis $\mathrm{C}$ virus coinfection, a history of diabetes, black race and male gender are potential risk factors causing renal injury.

Kidney involvement has been demonstrated with chronic diseases like leprosy and tuberculosis $[37,38]$. Renal injury in these diseases can lead to AKI and further progress to CKD and manifests as proteinuria, haematuria, urinary concentration and acidification defects, acute tubular necrosis, diffuse proliferative lesion and amyloidosis. An another common cause of renal injury in children in most tropical countries is Haemolytic uraemic syndrome (HUS). In India $35-41 \%$ of all cases of AKI in children are due to HUS [39]. Most common organism responsible for HUS in tropical countries includes Shigella dysenteriae in comparison to Verotoxin producing E.Coli from western countries. Schistosomiasis is an another tropical parasitic infection that can lead to kidney injury in the form of immune complex glomerulonephritis, also known as schistosomal nephropathy [40]. Fungal infections like renal mucormycosis caused by zygomycetes fungi can lead to kidney injury. Fungi invades blood vessels leading to thrombosis which causes infarction and necrosis of the organ involved. Mostly it involves bilateral kidneys and present with anuric AKI and resulting in a fatal outcome. Melioidosis is caused by pseudomonas pseudomallei encompassing a broad spectrum of disease processes. $61 \%$ of patients with acute septicaemic melioidosis have some component of renal injury, as observed in a study [41]. Yellow fever is a viral disease endemic to tropical Africa, South America, and Panama and has not been reported in India. It may be asymptomatic, may cause moderate febrile disease, or may cause haemorrhagic fever, liver failure, AKI, and death. Kidney injury mainly occurs in the form of acute tubular necrosis and may lead to increased mortality. Visceral leishmaniasis also known as kala azar is a chronic lethal parasitic disease, caused by Leishmania parasite. Chronic leishmaniasis usually has renal involvement which adds to the mortality. Patients usually present with proteinuria, microscopic haematuria and leukocyturia [42]. Renal involvement manifests in various forms like AKI, urinary concentration and acidification defect, nephritic syndrome or nephrotic syndrome.

\section{Eonclusion}

Kidney involvement in tropical countries is a challenging problem as it increases morbidity and mortality many folds. Symptomatic renal involvement in Tropical infectious disease signifies major organ dysfunction and is indirect reflection of severity of disease. The situation in developing countries is worsened due to the inadequacy of resources and patients presenting late to heath care centers. Coordinated and integrated approach from the primary to tertiary health care facilities is very crucial and can reduce the disease burden. Monitoring to assess renal injury, control of preventable risk factors and timely intervention with appropriate antibiotics $\&$ early use of renal replacement therapy once kidney injury have proven effective in preventing the long term complications. Patient education, healthy environmental conditions and improved health care facilities in the form of haemodialysis centres \& critical care facilities are a must requirement to fasten the health care in tropical areas. 


\section{References}

1. James Cook University. (2014). State of the Tropics - 2014 Report: James Cook University. Toensville.

2. Githeko, A.K., Ototo, E.N. \& Guiyun, Y. (2012). Progress towards understanding the ecology and epidemiology of malaria in the western Kenya highlands: opportunities and challenges for control under climate change risk. Acta Trop. 121(1), 19-25. doi: 10.1016/j. actatropica.2011.10.002.

3. Gomes, A.F., Nobre, A.A. \& Cruz, O.G. (2012). Temporal analysis of the relationship between dengue and meteorological variables in the city of Rio de Janeiro, Brazil, 2001-2009. Cad Saude Publica. 28(11), 2189-2197.

4. Basu, G., Chrispal, A., Boorugu, H., Gopinath, K.G., Chandy, S., Prakash, J.A., Thomas, K., Abraham, A.M. \& John, G.T. (2011). Acute kidney injury in tropical acute febrile illness in a tertiary care centre--RIFLE criteria validation. Nephrol Dial Transplant. 26(2), 524-531. doi: 10.1093/ndt/gfq477.

5. Jayakumar, M., Prabahar, M.R., Fernando, E.M., Manorajan, R., Venkatraman, R. \& Balaraman, V. (2006). Epidemiologic trend changes in acute renal failure--a tertiary center experience from South India. Ren Fail. 28(5), 405-410. doi: 10.1080/08860220600689034.

6. Chugh, K.S., Sakhuja, V., Malhotra, H.S. \& Pereira, B.J. (1989). Changing trends in acute renal failure in third-world countries-Chandigarh study. QJ Med. 73(272), 1117-1123.

7. Bellomo, R., Ronco, C., Kellum, J.A., Mehta, R.L., Palevsky, P. \& Acute Dialysis Quality Initiative, w. (2004). Acute renal failure definition, outcome measures, animal models, fluid therapy and information technology needs: the Second International Consensus Conference of the Acute Dialysis Quality Initiative (ADQI) Group. Crit Care. 8(4), R204-212. doi: 10.1186/ cc2872

8. Breman, J.G., Stekete, R.W. (1992). Malaria In:
Last, J.M., Wallace, R.B. (eds). Public Health and Preventive Medicine, 13th Ed. Norwalk, Conn Appleton Lange (pp 240-53).

9. Mandell, G.L., Bennett, J.E. \& Dolin, R. (2010). Mandell, Douglas, and Bennett's principles and practice of infectious diseases: Churchill Livingstone/Elsevier.

10. Mishra, S.K. \& Das, B.S. (2008). Malaria and acute kidney injury. Semin Nephrol. 28(4), 395408. doi: 10.1016/j.semnephrol.2008.04.007

11. Kaushik, R., Kaushik, R.M., Kakkar, R., Sharma, A. \& Chandra, H. (2013). Plasmodium vivax malaria complicated by acute kidney injury: experience at a referral hospital in Uttarakhand, India. Trans R Soc Trop Med Hyg. 107(3), 188194. doi: $10.1093 / \mathrm{trstmh} / \mathrm{trs} 092$

12. Wilairatana, P., Looareesuwan, S. \& Charoenlarp, P. (1994). Liver profile changes and complications in jaundiced patients with falciparum malaria. Trop Med Parasitol. 45(4), 298-302

13. Sitprija, V. (1988). Nephropathy in falciparum malaria. Kidney Int. 34(6), 867-877.

14. Barsoum, R.S. (1998). Malarial nephropathies. Nephrol Dial Transplant. 13(6), 1588-1597.

15. Mohanty, S., Mishra, S.K., Pati, S.S., Pattnaik, J. \& Das, B.S. (2003). Complications and mortality patterns due to Plasmodium falciparum malaria in hospitalized adults and children, Rourkela, Orissa, India. Trans R Soc Trop Med Hyg. 97(1), 69-70..

16. Barsoum, R.S. (2000). Malarial acute renal failure. J Am Soc Nephrol. 11(11), 2147-2154.

17. Lima, E.Q. \& Nogueira, M.L. (2008). Viral hemorrhagic fever-induced acute kidney injury. Semin Nephrol. 28(4), 409-415. doi: 10.1016/j. semnephrol.2008.04.009.

18. Lee, I.K., Liu, J.W. \& Yang, K.D. (2009). Clinical characteristics, risk factors, and outcomes in adults experiencing dengue hemorrhagic fever complicated with acute renal failure. Am J Trop Med Hyg. 80(4), 651-655.

19. Kuo, M.C., Lu, P.L., Chang, J.M., Lin, M.Y., Tsai, J.J., Chen, Y.H., Chang, K., Chen, H.C. \& Hwang, S.J. (2008). Impact of renal failure on the outcome of dengue viral infection. Clin $J$ Am Soc Nephrol. 3(5), 1350-1356. doi: 10.2215/ CJN.00020108. 
20. Mehra, N., Patel, A., Abraham, G., Reddy, Y.N. \& Reddy, Y.N. (2012). Acute kidney injury in dengue fever using Acute Kidney Injury Network criteria: incidence and risk factors. Trop Doct. 42(3), 160-162. doi: 10.1258/td.2012.120023.

21. Lima, E.Q., Gorayeb, F.S., Zanon, J.R., Nogueira, M.L., Ramalho, H.J. \& Burdmann, E.A. (2007). Dengue haemorrhagic fever-induced acute kidney injury without hypotension, haemolysis or rhabdomyolysis. Nephrol Dial Transplant. 22(11), 3322-3326. doi: 10.1093/ndt/gfm431

22. Toliver, H.L. \& Krane, N.K. (2014). Leptospirosis in New Orleans. Am J Med Sci. 347(2), 159-163. doi: 10.1097/MAJ.0b013e3182787068.

23. Andrade, L., de Francesco Daher, E. \& Seguro, A.C. (2008). Leptospiral nephropathy. Semin Nephrol. 28(4), 383-394. doi: 10.1016/j. semnephrol.2008.04.008

24. Sitprija, V., Losuwanrak, K. \& Kanjanabuch, T. (2003). Leptospiral nephropathy. Semin Nephrol. 23(1), 42-48. doi: 10.1053/snep.2003.50004

25. Seguro, A.C., Lomar, A.V. \& Rocha, A.S. (1990). Acute renal failure of leptospirosis: nonoliguric and hypokalemic forms. Nephron. 55(2), 146151.

26. Panaphut, T., Domrongkitchaiporn, S., Vibhagool, A., Thinkamrop, B. \& Susaengrat, W. (2003). Ceftriaxone compared with sodium penicillin $\mathrm{g}$ for treatment of severe leptospirosis. Clin Infect Dis. 36(12), 1507-1513. doi: 10.1086/375226.

27. Seong, S.Y., Choi, M.S. \& Kim, I.S. (2001). Orientia tsutsugamushi infection: overview and immune responses. Microbes Infect. 3(1), 11-21.

28. Aggarwal, H., Jain, D., Kaverapp, V., Mittal, A., Yadav, S. \& Gupta, A. (2015). Emergence of Scrub Typhus in Northern India: Experience from Tertiary Care Hospital. Klimik Dergisi. 27(1), 6-11. doi: 10.5152/kd.2014.03.

29. Tsay, R.W. \& Chang, F.Y. (1998). Serious complications in scrub typhus. $J$ Microbiol Immunol Infect. 31(4), 240-244.

30. Yen, T.H., Chang, C.T., Lin, J.L., Jiang, J.R. \& Lee, K.F. (2003). Scrub typhus: a frequently overlooked cause of acute renal failure. Ren Fail. 25(3), 397-410.

31. Daher Ede, F., da Silva, G.B., Jr. \& Barros, E.J. (2013). Renal tuberculosis in the modern era.
Am J Trop Med Hyg. 88(1), 54-64. doi: 10.4269/ ajtmh.2013.12-0413.

32. Eastwood, J.B., Corbishley, C.M. \& Grange, J.M. (2001). Tuberculosis and the kidney. $J$ Am Soc Nephrol. 12(6), 1307-1314..

33. Figueiredo, A.A., Lucon, A.M., Arvellos, A.N., Ramos, C.O., Toledo, A.C., Falci, R., Jr., Gomes, C.M., Recaverren, F.E., Netto, J.M. \& Srougi, M. (2010). A better understanding of urogenital tuberculosis pathophysiology based on radiological findings. Eur J Radiol. 76(2), 246-257. doi: 10.1016/j.ejrad.2009.05.049.

34. Muttarak, M., ChiangMai, W.N. \& Lojanapiwat, B. (2005). Tuberculosis of the genitourinary tract: imaging features with pathological correlation. Singapore Med J. 46(10), 568-574; quiz 575.

35. Chugh, K.S., Narang, A., Kumar, L., Sakhuja, V., Unni, V.N., Pirzada, R., Singh, N., Pereira, B.J. \& Singhal, P.C. (1987). Acute renal failure amongst children in a tropical environment. Int $J$ Artif Organs. 10(2), 97-101.

36. Aggarwal, H.K., Jain, D., Dahiya, S., Jain, P. \& Pawar, S. (2015). Evaluation of renal profile in asymptomatic HIV patients with special reference to proteinuria. Medical Studies/ Studia Medyczne. 30(4), 228-233. doi: 10.5114/ ms.2014.47920.

37. Daher, E.F., Silva, G.B., Jr., Cezar, L.C., Lima, R.S., Gurjao, N.H., Mota, R.M., Abreu, K.L., Rocha, N.A., Oliveira, M.J. \& Liborio, A.B. (2011). Renal dysfunction in leprosy: a historical cohort of 923 patients in Brazil. Trop Doct. 41(3), 148-150. doi: 10.1258/td.2011.100436.

38. Eastwood, J.B., Corbishley, C.M. \& Grange, J.M. (2001). Tuberculosis and the kidney. $J$ Am Soc Nephrol. 12(6), 1307-1314.

39. Srivastava, R.N., Bagga, A. \& Moudgil, A. (1990). Acute renal failure in north Indian children. Indian J Med Res. 92, 404-408..

40. Barsoum, R.S. (2013). Parasitic kidney disease: milestones in the evolution of our knowledge. Am J Kidney Dis. 61(3), 501-513. doi: 10.1053/j. ajkd.2012.09.025.

41. Susaengrat, W., Dhiensiri, T., Sinavatana, P. \& Sitprija, V. (1987). Renal failure in melioidosis. Nephron. 46(2), 167-169.

42. Agenor Araujo Lima Verde, F., Araujo Lima 
Verde, F., De Francesco Daher, E., Martins Dos Santos, G., Saboia Neto, A. \& Mendoca Lima Verde, E. (2009). Renal tubular dysfunction in human visceral leishmaniasis (Kala-azar). Clin Nephrol. 71(5), 492-500. 